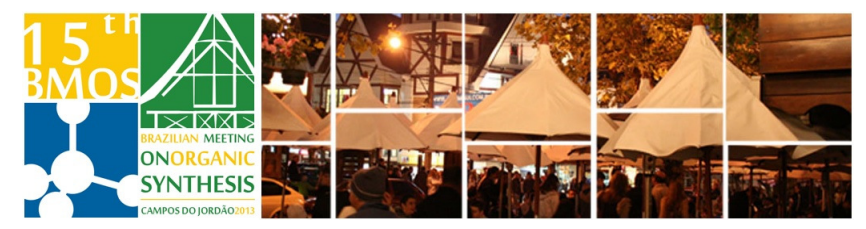

\title{
Microwave-assisted synthesis of fused tetrazole- ketopiperazine compounds via Ugi reactions
}

\author{
André Felipe C. Amaral, Wender A. Silva, Carlos Kleber Z. Andrade*
}

Laboratório de Química Metodológica e Orgânica Sintética (LaQMOS), IQ-UNB.

Keywords: Tetrazole, ketopiperazine, Ugi reaction

\section{INTRODUCTION}

Multicomponent Reactions (MCRs) offer many advantages, and due to their ability to rapidly create molecular diversity and molecular complexity, allowing the generation of compounds containing essentially all the atoms of the reactants, they have become an important way to synthesize novel bioactive products. ${ }^{1}$

The classical Ugi four-component $(\mathrm{U}-4 \mathrm{CR})^{2}$ reaction is one of the most known multicomponent reaction, in which an isocyanide, an aldehyde or ketone, a carboxylic acid and an amine combine to yield $\alpha$ acylamino amides. We report herein a concise way to synthezise compounds containing both the tetrazole and ketopiperazine nuclei as synergistic moieties via a modified Ugi reaction in which the acid has been replaced by trimethylsilylazide $\left(\mathrm{TMSN}_{3}\right)$. Tetrazoles and ketopiperazines are important heterocycles and both have interest in the pharmaceutical industry. ${ }^{3}$

\section{RESULTS AND DISCUSSION}

We began the synthesis by the initial in situ formation of the imine (MW, $10 \mathrm{~min}, 100 \stackrel{\circ}{\circ} \mathrm{C}$ ) followed by its reaction with the isocyanide and trimethylsilylazide (MW, $10 \mathrm{~min}, 120^{\circ} \mathrm{C}$ ), Scheme 1. This Ugi-3CR process gave better results when compared to the Ugi-4CR. Then, the expected tetrazolic products 1a-h were subjected, without isolation, to the reaction with trifluoroacetic acid under microwave irradiation for 10 minutes at 120 ${ }^{\circ} \mathrm{C}$. All reactions were realized in a microwave reactor (CEM Co, Discover).

The final products $2 \mathrm{a}-\mathrm{e}$ were obtained in a very clean process and purified by column chromatography, using 30\% EtOAc in hexane, yielding the pure products in good yields over the three steps.

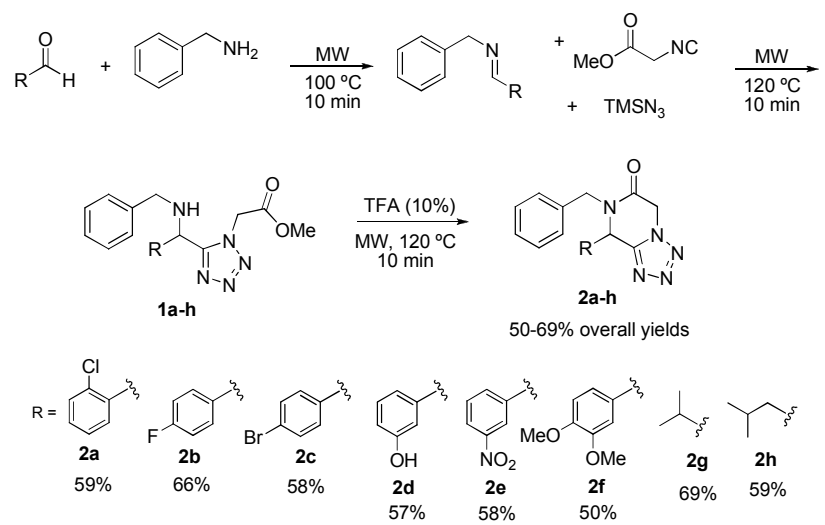

Scheme 1. Synthesis of fused tetrazole-ketopiperazine compounds.

\section{CONCLUSION}

The proposed methodology represents an efficient and clean way to synthesize tetrazoleketopiperazines via a modified Ugi-3CR reaction followed by acid catalyzed cyclization. Eight new molecules cointaining both the tetrazolic and ketopiperazinic nuclei were obtained in very good overall yields, in short reaction times and in a solventless process.

\section{ACKNOWLEDGEMENTS}

IQ-UnB, CAPES, CNPq and FINEP-CTINFRA nํ0 0970/01.

\section{REFERENCES}

${ }^{1}$ a) Dömling, A.; Ugi, I.; Angew. Chem. Int. Ed. 2000, 39, 3169. b) Dömling, A.; Chem. Rev. 2006, 106, 17.

${ }^{2}$ Ugi I.; Fetzer, I.; Steinbrückner, C.; Angew. Chem. 1959, 71, 386.

${ }^{3}$ Katritzky, A. R.; Ramsden, C. A.; Scriven, E. F. V.; Taylor, R. J. K., Eds. In Comprehensive Heterocyclic Chemistry III, 2008, Elsevier. 